\title{
Guideline for Test Process Improvement of Test Organization Through Correlating TMMi with TPI NEXT
}

\author{
Kidu Kim ${ }^{\dagger} \cdot$ Park Young Bom ${ }^{++} \cdot$ R. Youngchul Kim ${ }^{+++}$
}

\begin{abstract}
In this paper, it will improve in quality to adapt a right test process which is formalized from certification of TMMi Level. To do this, we suggest correlative relation through analyzing associations between TMMi and TPI next based on the previous research[10], which provides the guideline for enhancing test process level with measuring Test maturity model. Also schematize test maturity measurement through refining and improving the previous test maturity correlation metrics[6,8,9]. As one example with limited level, it shows the guideline to improve test process of one testing organization through improved correlation metrics with TMMi and TPI next.
\end{abstract}

Keywords : TMMi(Test Maturity Model Integration), TPI Next(Test Process Improvement), Test Maturity Correlation Metrics

\section{상관관계를 통한 조직의 테스트 프로세스 개선 가이드 방안}

\author{
김 기 두 ${ }^{\dagger}$ 박 용 범 ${ }^{+\dagger} \cdot$ 김 영 철 ${ }^{+\dagger}$
}

\begin{abstract}
요 약
본 논문에서는 기본적인 TMMi 레벨 인증으로부터 정형화된 올바른 테스트 프로세스를 적용하여 소프트웨어의 품질을 높이고자 한다. 조직 의 테스트 성숙도 수준 평가 모델인 TMMi과 테스트 프로세스를 개선 모델인 TPI NEXT의 분석을 통해 상관 관계도를 제안하고, 그 기반에서 조직의 테스트 성숙도 측정으로 테스트 프로세스 향상을 위한 가이드를 제공하고자 한다. 또한 기존의 테스트 성숙도 상호관련 메트릭의 보완 및 개선 통해 성숙도 측정을 도식화 한다. 제한된 레벨 수준의 사례로 TMMi와 TPI NEXT의 상관 관계도와 보완한 테스트 성숙도 상호관련 메트릭을 통해 조직의 테스트 프로세스 개선을 위한 가이드를 보여준다.
\end{abstract}

키워드 : 테스트 성숙도 모델(TMMi), 테스트 프로세스 개선 모델(TPI NEXT), 테스트 성숙도 상호관련 메트릭스

\section{1. 서 론}

정보통신 분야의 발전으로 인해 많은 제품의 소프트웨어 들이 개발되어 있다. 그로 인해 소프트웨어간의 품질 차이 를 경험하게 되었다. 하지만, 사용자 혹은 고객들은 항상 높 은 품질 수준의 소프트웨어들을 사용하고자 한다. 이에 맞 춰 개발자들은 높은 품질 수준의 소프트웨어를 개발해야한 다. 높은 품질 수준의 소프트웨어를 개발하기 위해서는 완 벽한 테스트를 수행해야하지만 실행할 수 없다. 이를 위해 사용할 수 있는 것이 CMMi, TMMi, TPI next 등의 소프트

\footnotetext{
\% 이 논문은 본 연구는 미래창조과학부 및 한국산업기술평가관리원의 산업 원천기술개발사업[10035708, 고신뢰 자율제어 SW를 위한 CPS(CyberPhysical Systems) 핵심 기술 개발]과 2013년도 정부(교육부)의 재원으로 한국연구재단의 지원을 받아 수행된 기초연구사업임(2013R1A1A2011601).

† 정 회 원 : 한국정보통신기술협회 소프트웨어시험인증연구소 선임연구원

†† 종신회원 단국대학교 컴퓨터과학과 교수

††† 정 회 원 : 홍익대학교 컴퓨터정보통신 교수 논문접수 : 2013년 7월 1일

수 정 일 : 1 차 2013년 8월 23일

심사완료 : 2013년 8월 24일

* Corresponding Author:R. Youngchul Kim(bob@hongik.ac.kr)
}

웨어 평가 모델들이 존재한다. 국내에는 이미 TMMi 평가를 받은 조직들이 있는데, 이들이 테스트 프로세스를 평가 받 고 개선하기 위해서는 TPI next와 같은 프로세스 개선 모 델의 추가 심사를 받아야한다.

본 논문에서 이를 보완하기 위해 TMMi와 TPI next 모 델간 분석을 통해 비/공통 요소 및 상관 관계를 도출하여 TMMi 모델만 적용하여도 TPI next 모델의 수준을 파악할 수 있으며, 나아가 조직의 테스트 프로세스를 개선할 수 있 도록 지원하고자 한다. 본 논문 1장에서는 연구 내용 개요, 2장에서는 비교 대상인 TMMi와 TPI next 모델 소개, 3장 에서는 모델 비교에 따른 비/공통 요소 및 상관관계 도출 방법 제시, 4장에서는 테스트 프로세스 개선 사례 소개, 5장 에서는 연구 결과 및 추후 의 구조로 기술되었다.

\section{2. 관련 연구}

최초 테스트 성숙도 모델(TMM)[3,4,5]은 일리노이 공대 의 Burnstein 교수 팀에 의해 테스트 성숙도를 측정을 목적 
으로 개발된 모델이다. 테스트 성숙도 모델은 1996년 능력 성숙도 모델(CMM)에서 테스트 활동에 대한 프로세스 개선 을 보조하기 위해 개발되었으며, 기존의 심사 모델 중에서 도 가장 일관성 있고 완전한 성숙도 모델 구조를 갖고 있으 며, 심사 모델 및 절차, 심사 모델 및 절차, 심사 도구 및 질 문서, 팀 교육 등에 관한 기준을 제시하고 있다. 현재는 기 존의 TMM에 여러 테스트 모델들이 통합된 TMMi(Test Maturity Model Integration)이 개발되어 있다[1].

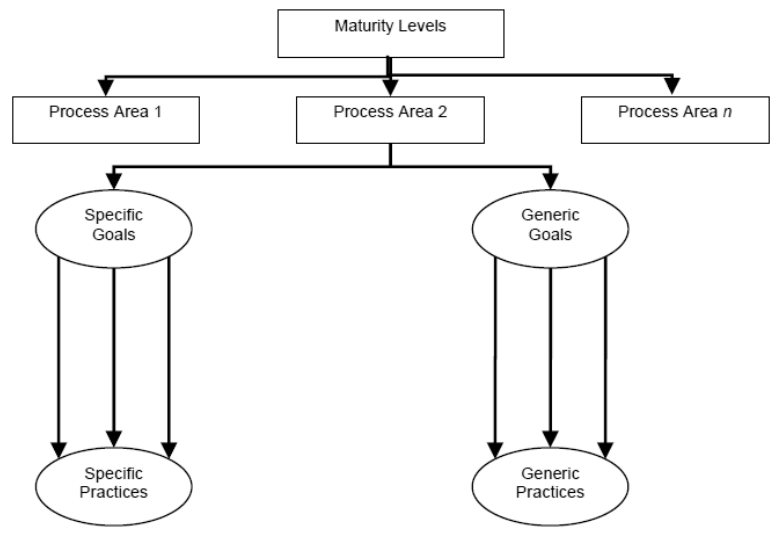

Fig. 1. TMMi Framework[1]

테스트 성숙도 모델은 Fig. 1의 구조도에 나타난 것과 같 이 테스팅 능력을 나타내는 레벨들이 존재하며, 각 성숙도 레벨은 모두 5 개의 레벨로 정의하고 있다. 또한, 레벨은 각 각의 레벨에서 수행되어야하는 성숙도 목표를 포함하고 하 며, 성숙도 목표는 하부 목표를 갖고 있다. TMMi는 테스트 성숙도를 각 레벨별로 정의하고 있으며, 각각의 성숙도 레 벨에 대한 프로세스 영역들이 있다. 또한, 아래 Table 1과 같이 각 영역에서 수행해야할 상세 목표와 일반 목표로 구 성되어 있다.

기존의 TPI(Test Process Improvement) 모델은 테스트 프로세스 개선을 보다 쉽게 수행하기 위해 1997년 Tim Koomen과 Martin Pol에 의해 개발된 모델이다. TPI의 특징 은 조직의 현재 조직의 테스트 프로세스의 강점과 약점을 파악하여 체크포인트를 통해 프로세스 성숙도를 평가하고, 개선 사항을 제시하고 있다.

TPI NEXT는 기존의 테스트 프로세스 개선 모델(TPI)을 확장하여 현재는 웹기반의 비즈니스 적용에 용이하게 개선 하였다. TPI NEXT의 구조를 살펴보면 테스트 프로세스에 서 관련 기술, 도구, 보고서등을 나타내는 핵심영역(Key Areas), 각 핵심영역을 시험하고 성숙도 수준을 분류하는 성속도 레벨(Maturity Levels), 모든 핵심영역을 통해 결정 되는 테스트 프로세스의 수준을 보여주는 테스트 성숙도 메 트릭(Test Maturity Metrics), 핵심영역을 객관적으로 평가 할 수 있는 체크 포인트(Check Point), 측정된 수준보다 테 스트 프로세스를 향상시키기 위한 개선 제안(Improvement Suggestion), 여러 핵심영역(Key Areas)에서의 체크포인트 집합인 클러스터(Clusters), 테스트 프로세스의 개선을 유지
Table 1. TMMi(Level 2)

\begin{tabular}{|c|c|c|}
\hline Level & PA(Process Area) & SG(Specific Goal) \\
\hline \multirow{18}{*}{ Level2 } & \multirow{3}{*}{$\begin{array}{l}\text { PA2.1 Test Policy } \\
\text { and Strategy }\end{array}$} & SG1 Establish a Test Policy \\
\hline & & SG2 Establish a Test Strategy \\
\hline & & $\begin{array}{c}\text { SG3 Establish test Performance } \\
\text { Indicators }\end{array}$ \\
\hline & \multirow{5}{*}{ PA2.2 Test Planning } & $\begin{array}{c}\text { SG1 Perform a Product Risk } \\
\text { Assessment }\end{array}$ \\
\hline & & SG2 Establish a Test Approach \\
\hline & & SG3 Establish Test Estimates \\
\hline & & SG4 Develop a Test Plan \\
\hline & & $\begin{array}{l}\text { SG5 Obtain Commitment to The Test } \\
\text { Plan }\end{array}$ \\
\hline & \multirow{3}{*}{$\begin{array}{l}\text { PA2.3 Monitoring } \\
\text { and Control }\end{array}$} & $\begin{array}{c}\text { SG1 Monitor Test Progress Against } \\
\text { Plan }\end{array}$ \\
\hline & & $\begin{array}{l}\text { SG2 Monitor Product Quality Against } \\
\text { Plan and Expectations }\end{array}$ \\
\hline & & $\begin{array}{c}\text { SG3 Manage Corrective Action to } \\
\text { Closure }\end{array}$ \\
\hline & \multirow{4}{*}{$\begin{array}{l}\text { PA2.4 Test Design } \\
\text { and Execution }\end{array}$} & $\begin{array}{c}\text { SG1 Perform Test Analysis and } \\
\text { Design Using Test Design Techniques }\end{array}$ \\
\hline & & SG2 Perform Test Implementation \\
\hline & & SG3 Perform Test Execution \\
\hline & & $\begin{array}{c}\text { SG4 Manage Test Incidents to } \\
\text { Closure }\end{array}$ \\
\hline & \multirow{3}{*}{$\begin{array}{l}\text { PA2.5 Test } \\
\text { Environment }\end{array}$} & $\begin{array}{c}\text { SG1 Develop Test Environment } \\
\text { Requirement }\end{array}$ \\
\hline & & $\begin{array}{l}\text { SG2 Perform Test Environment } \\
\text { Implementation }\end{array}$ \\
\hline & & $\begin{array}{c}\text { SG3 Manage and Control Test } \\
\text { Environment }\end{array}$ \\
\hline
\end{tabular}

하기 위해 소프트웨어 생명주기와 다른 프로세스들을 동조 시키는 원동력은(Enablers)으로 구성된다[2].

테스트 프로세스 개선모델(TPI NEXT)은 테스트 성숙도 모델(TMMi)과 달리 레벨 평가뿐만 아니라 TMMi 보다 적 극적으로 테스트 프로세스를 개선하도록 유도하고 있다. TPI NEXT 모델은 테스트 프로세스의 수준 확인을 위한 항 목인 16개의 키영역(Key Area)과 현재 테스트 프로세스 수 준을 구분하기 위한 4개의 성숙도 수준(Maturity Levels)으 로 구성되어 있다. TPI NEXT의 핵심영역과 성숙도 수준은 아래 Fig. 2와 같다.

아래 Table 2와 같이 TMMi 모델은 질의서를 통해 평가 하며, 성숙도 레벨이 1 5까지 모두 5단계이다. 또한, 기존의 $\mathrm{CMMI}, \mathrm{ISO}, \mathrm{SPICE}, \mathrm{ISTQB}$ 을 통해 기존의 $\mathrm{TMM}$ 을 확장 하였다. 반면 TPI NEXT의 경우 프로세스 평가를 위한 체 크리스트를 이용하며, Initial Optimizing까지 4단계의 레벨 로 이루어져 경험 기반의 모델이다. 


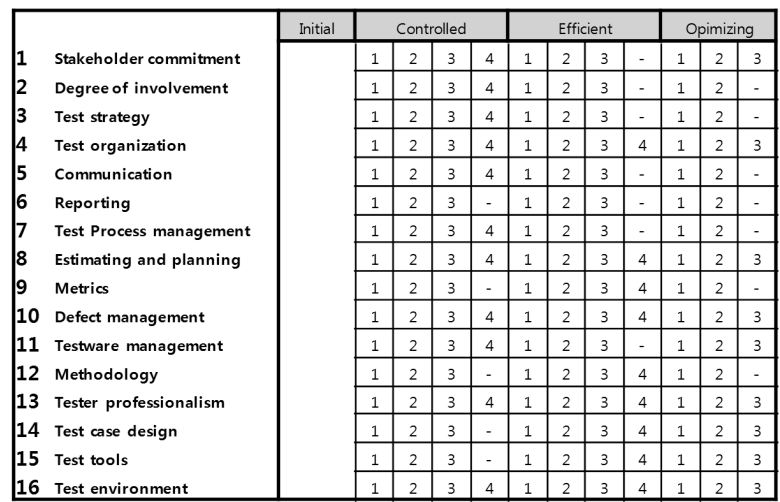

Fig. 2. TPI NEXT Matrix

Table 2. TMMi vs TPI NEXT

\begin{tabular}{|c|c|c|}
\hline Model & TMMi & TPI NEXT \\
\hline Development & 2008 & 2009 \\
\hline Feature & Conceptual Model & Experience Model \\
\hline Organization & TMMi Foundation & Sogeti \\
\hline Level & 5 & 4 \\
\hline KPA & 10 & 16 \\
\hline Evaluation Method & Questionnaire & Checklist \\
\hline Based Model & $\begin{array}{c}\text { CMMI, ISO, SPICE, } \\
\text { ISTQB }\end{array}$ & Experience-based \\
\hline
\end{tabular}

\section{3. 조직의 테스트 프로세스 향상}

본 연구에서는 TMMi와 TPI NEXT의 비/공통요소들을 통해 테스트 프로세스를 개선하고자 한다. 이를 위해 우선적 으로 $\mathrm{TMMi}$ 와 TPI NEXT 항목들에 대한 비교 분석이 필요 하다. 우리는 두 모델이 갖고 있는 항목들을 비교 분석하여 아래 Fig. 3과 같이 모델간 매핑이 가능함을 알 수 있었다.

- Step 1: TMMi(Level2)과 TPI NEXT 매핑

TPI NEXT와 TMMi Framework의 경우 두 모델에서 제 시하는 수준(Level)의 목표가 유사함을 Fig. 4에서 알 수 있

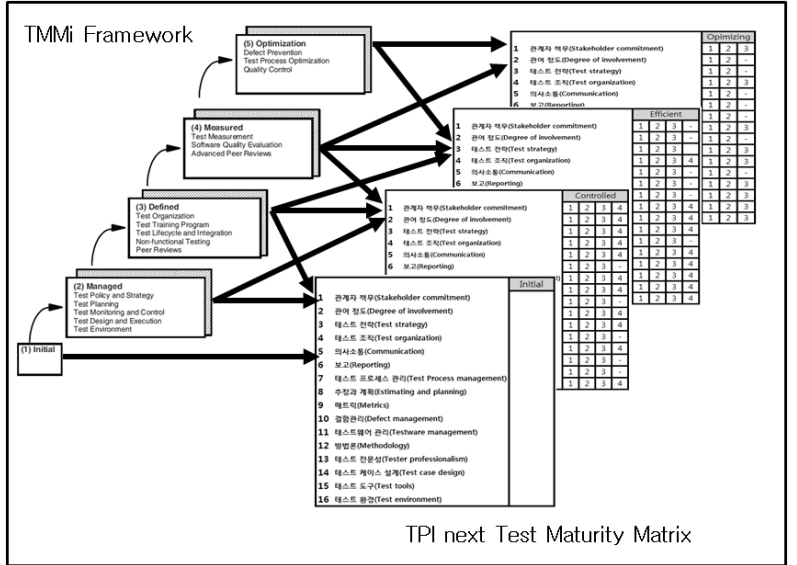

Fig. 3. TMMi Framework and TPI NEXT Correlation
었다. 이를 기반으로 TPI NEXT 핵심영역(Key Area)과 TMMi의 Specific Goal의 매핑을 통한 연관성분석으로 비/ 공통 요소를 추출한다. 각 모델간의 매핑은 매핑 항목이 의 미하는 바를 프로젝트 수행 경험을 기반으로 분석하였다. 예를 들면, TPI NEXT의 '3. 테스트 전략'과 TMMi의 'SP3, 테스트 이해관계자에게 테스트 전략 배포'의 연관성을 분석 하여 유사 항목임을 발견할 수 있었다. 동일한 방법으로 TMMi(Level2)의 항목을 대상으로 두 모델간의 분석결과는 아래 Fig. 4와 같다.

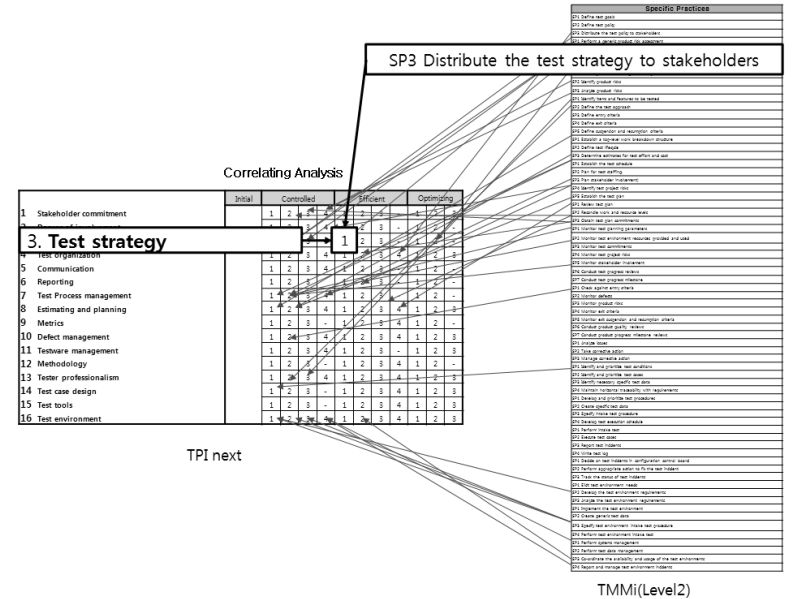

Fig. 4. Comparison of TPI NEXT and TMMi(Level 2)

- Step 2 : 비/공통 요소 식별

앞에서 우리는 TMMi(Level2)와 TPI NEXT의 요소 간 매핑이 가능하였으며, Step 1의 연관성 분석을 통해 비/공통 요소를 찾을 수 있었다. 비공통 요소들은 TPI NEXT 혹은 TMMi(level2)가 서로 갖고 있지 않는 요소들로 Fig. 4에서 화살표로 연결되지 않는 부분들이다. 그리고, 공통요소들은 연관성 분석을 통해 두 모델이 공통으로 갖고 있는 영역로 Fig. 4에서 화살표로 연결된 부분들이다. 두 모델에서 식별 된 비/공통 요소를 정리하면 아래 Table 3과 같다.

Table 3. Un/Common Items

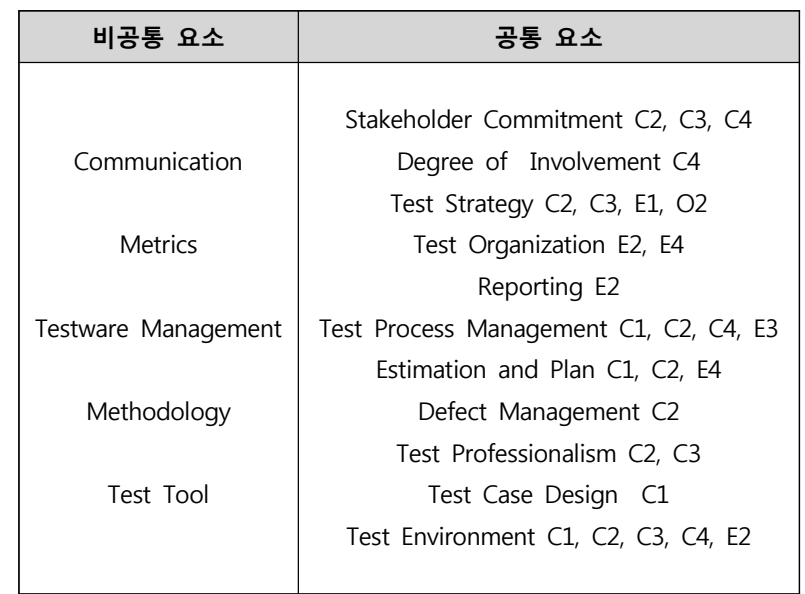


- Step 3 : 테스트 프로세스 보완

Step 1, 2를 통해 추출된 비/공통 요소들은 Fig. 6 과 같이 각 영역들이 빠짐 없이 $1: 1$ 로 매핑되지 않는다. 그로 인해 TMMi의 Specific Goal은 TPI NEXT의 성숙도 수준 (Controlled, Efficient, Optimizing)에 내포된 등급(scale)에 모두 매핑되지 않는다. 이를 위해 우리는 등급과 등급 간이 2 단계 이상 차이가 생길 경우 이전 등급을 실제 프로세스 성숙도 수준으로 판단하였다. 예를 들면, 테스트 전략(Test Strategy)의 경우 TMMi(level2)에서는 Optimizing 수준의 2 등급의 요소도 포함하고 있지만 Efficient의 등급과 3 단계 차이가 발생하기 때문에 TPI NEXT의 Efficient 등급 1 을 만족한다고 판단하였다.

\begin{tabular}{|c|c|c|c|c|c|c|c|c|c|c|c|c|}
\hline \multirow[b]{2}{*}{ Stakeholder commitment } & \multirow[t]{2}{*}{ Initial } & \multicolumn{4}{|c|}{ Controlled } & \multicolumn{4}{|c|}{ Efficient } & \multicolumn{3}{|c|}{ Optimizing } \\
\hline & & 1 & & & & 1 & 2 & 3 & - & 1 & 2 & 3 \\
\hline 2 Degree of involvement & & 1 & 2 & 3 & $\Rightarrow$ & 1 & 2 & 3 & - & 1 & 2 & \\
\hline 3 Test strategy & & 1 & 2 & 3 & 4 & $\gg$ & 2 & 3 & - & 1 & 2 & - \\
\hline 4 Test organization & & 1 & 2 & 3 & 4 & 1 & 2 & 3 & $\rightarrow$ & 1 & 2 & 3 \\
\hline 5 Communication & & 1 & 2 & 3 & 4 & 1 & 2 & 3 & - & 1 & 2 & - \\
\hline 6 Reporting & & 1 & 2 & 3 & & 1 & $\Rightarrow$ & 3 & - & 1 & 2 & - \\
\hline 7 Test Process management & & 1 & 2 & -3 & $\Rightarrow$ & 1 & 2 & 3 & - & 1 & 2 & - \\
\hline 8 Estimating and planning & & 1 & $\Rightarrow$ & 3 & 4 & 1 & 2 & 3 & 4 & 1 & 2 & 3 \\
\hline 9 Metrics & & 1 & 2 & 3 & - & 1 & 2 & 3 & 4 & 1 & 2 & - \\
\hline 10 Defect management & & 1 & 2 & 3 & 4 & 1 & 2 & 3 & 4 & 1 & 2 & 3 \\
\hline 11 Testware management & & 1 & 2 & 3 & 4 & 1 & 2 & 3 & - & 1 & 2 & 3 \\
\hline 12 Methodology & & 1 & 2 & 3 & - & 1 & 2 & 3 & 4 & 1 & 2 & - \\
\hline 13 Tester professionalism & & 1 & 2 & $\nabla$ & 4 & 1 & 2 & 3 & 4 & 1 & 2 & 3 \\
\hline 14 Test case design & & $\gg$ & 2 & 3 & - & 1 & 2 & 3 & 4 & 1 & 2 & 3 \\
\hline 15 Test tools & & 1 & 2 & 3 & - & 1 & 2 & 3 & 4 & 1 & 2 & 3 \\
\hline 16 Test environment & & 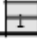 & 2 & 3 & 4 & 1 & 3 & 3 & 4 & 1 & 2 & 3 \\
\hline
\end{tabular}

Fig. 5. Mapping of TMMi(Level 2) and TPI NEXT

- Step 4 : 테스트 프로세스 향상

현재 TMMi 평가를 받은 레벨 2의 조직이 테스트 프로세 스 수준이 아래 그림과 같이 초기 단계('NOW')로 평가되었 을 경우 매핑 이후 부족한 비공통요소들만 개선한 다면 다 음 수준인 'Controlled'로 도달할 수 있다.

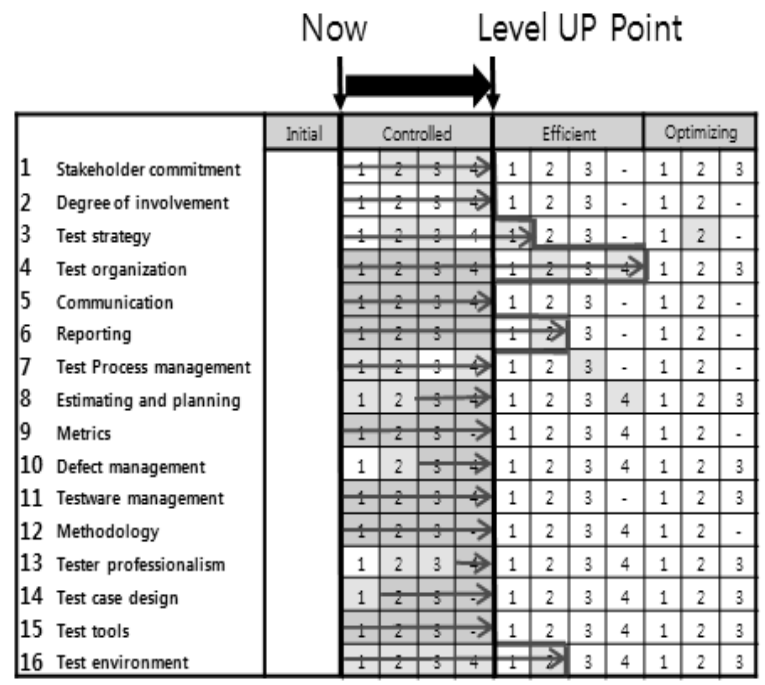

Fig. 6. Test Process Improvement

\section{4. 적용사례}

앞에서 추출된 비/공통 요소를 기반으로 기존의 테스트 성숙도 상호관련 메트릭(Test Attributes to Maturity Levels Correlation Metrics)[10]을 개선 및 확장하여 확장된 테스트 성숙도 상호관련 메트릭(Enhanced Test Attributes to Maturity Levels Correlation Metrics)를 제안한다. 확장 된 테스트 성숙도 상호관련 메트릭에는 TMMi에서 부족한 테스트 프로세스 항목들을 TPI NEXT의 비공통 요소를 통 해 개선할 수 있도록 하였다. 즉, 확장된 테스트 성숙도 상 호관련 메트릭를 통해 평가된 테스트 조직은 $\mathrm{TMMi}$ 평가 후에도 부족한 테스트 프로세스항목들에 대해 보완할 수 있 도록 제시하였다.

TTA 사례를 통해, 소수의 인원(5명)이 짧은 기간(약 3개 월) 동안 스캔프로그램을 개발하는 $\mathrm{A}$ 업체의 개발 조직을 선 정하였으며, 해당 조직의 테스트 성숙도를 평가한 결과 레 벨 1수준이었다. 이 조직을 다시 개선된 테스트 성숙도 상 호관련 메트릭을 통해 평가한 결과를 살펴보면 아래와 같 다. TS(Test Strategy), DOI(Degree of Involvement), TMT(Test Methodology), TT(Test Tools), TMEN(Test Mentoring), COM(Communication), RET(Reporting), TO (Test Organization)의 항목들의 경우 레벨2를 만족하였다. 특히, 테스트 다수의 테스트 도구를 갖고 테스트 프로젝트 에 진행하여 TT항목은 레벨 3까지 만족하였다.

하지만, 이와 반대로 $\mathrm{TPD}$ (Test Process Definition), TREV(Test Reviews), TPM(Test Process Management), TFI(Test Feedback Improvement), TMTC(Test Metrics), TSTD(Test Standards), TEAP(Test Estimating and planning), TST(Test Specification Techniques), TCD(Test Case Design), TDOC(Test Documents), TPDOC(Test Process Documents ), TE(Test Environment), TOE(Test Office Environment), TTRN(Test Training), DM(Defect Management), TWM(Testware Management), EVL(Evaluation), LLT(Low-level Testing), SC(Stakeholder Commitment), $\mathrm{TPF}$ (Tester Professionalism)에서 부족한 항 목들을 발견되어 레벨 1수준 이었다.

평가를 통해 발견된 부족한 항목으로 해당 조직의 프로세 스 약점을 분석할 수 있었다.(예: 정형화된 방법론을 정립해 두었지만 실제 프로젝트 진행에서의 활용도는 부족함 등) 또한, 앞서 수행한 평가 때 조직에서 만족하지 못했던 테스 트 성숙도 항목들을 Fig. 7과 같이 확장된 테스트 성숙도 상호관련 메트릭을 통해 테스트 프로세스의 수준을 향상 (level 1->level2)할 수 있도록 가이드(예: 테스트 수행에 따 른 결과물 관리가 이루어져야함, 테스트 수행 단계별 테스 터가 수행할 업무 정의가 필요함 등) 할 수 있었다.

\section{5. 결 론}

본 연구는 다양한 소프트웨어 테스트 관련 모델 중에서 


\begin{tabular}{|c|c|c|c|c|}
\hline \multicolumn{5}{|c|}{ Enhanced Test Attributes to Maturity Levels Correlation Metrics } \\
\hline 구표 & Test Atritute & L.VE| 1 & Lvตg| 2 & LขVI 3 \\
\hline \multirow{5}{*}{$\begin{array}{c}\text { Basic } \\
\text { TestProcess: }\end{array}$} & Test Process Defintion (TPD) & TPD 1 & TPD 2 & TPD 3 \\
\hline & Test Agvigwa (TRE)" & TREV/ 1 & TRE $/ 2$ & TRE/ 3 \\
\hline & Test 8tategy (TB) & T3 1 & T3 2 & I 783 \\
\hline & Degese of invovement $(D O C)$ & Do11 & 0012 & 0013 \\
\hline & Test Procses (Mnnessmennt(TPM) & ТРМ 1 & TPM 2 & TPM 3 \\
\hline \multirow[t]{3}{*}{ TestQuality. } & Test Fasodeck improvementitifi) & TFI 1 & TF 2 & $T F / 3$ \\
\hline & Test Natrica (Tinc) & TITC 1 & TITC 8 & TITC 3 \\
\hline & Teat standerca (TדTD) & TSTO & T980 2 & TSTO 3 \\
\hline \multirow{4}{*}{$\begin{array}{c}\text { Test } \\
\text { Tectriques }\end{array}$} & Test Estlmating and pesnning(TEAP) & EAP 1 & EAP ? & EAP 2 \\
\hline & Test 80ocillcation Teccmlavas (TST) & Тв 1 & твт 1 & Төт 8 \\
\hline & Test Matnoodooy (TIMT) & TוTI & Tiा\% & Thrт \\
\hline & TeAt Case Design(TCD) & $T 001$ & TCD2 & $\mathrm{TCD} 3$ \\
\hline \multirow[t]{2}{*}{ TestDocunent: } & Teat Documenta (TDOC) & $\mathrm{TOOCO} 1$ & ToOC 2 & $T 0003$ \\
\hline & Test Procsas Documgnta (TPDOC) & TPDOC 1 & TPDOC 2 & TPDOC 3 \\
\hline \multirow{3}{*}{$\begin{array}{c}\text { Test } \\
\text { Infrastucire } \\
\text { and took }\end{array}$} & Test Toosis(T) & $\pi 1$ & $\pi 1$ & $\pi 2$ \\
\hline & Test Environment(TE) & TE 1 & TE 2 & TE 3 \\
\hline & Teat OHllas Environment(TOE) & TOE 1 & TOE 1 & TOE 2 \\
\hline \multirow[t]{2}{*}{ TestEducaion: } & Test Trahling (TRAN) & TREN 1 & $\operatorname{TTHN} 2$ & TRAN 3 \\
\hline & Test IMantoling (TMINEN) & THEN 1 & THEN 1 & THEN 2 \\
\hline \multirow{9}{*}{$\begin{array}{c}\text { Test } \\
\text { Organization }\end{array}$} & Communication(COM) & $\cos 1$ & $\operatorname{col} 2$ & $\cos 3$ \\
\hline & Agooting(RET) & AET 1 & AET 2 & AET 3 \\
\hline & Detect Lenespementi(DWI) & DAM & DIN 2 & 0143 \\
\hline & Testwerg Menepgment(TWMi) & Twa 1 & TNM 2 & Twa 3 \\
\hline & Evaluation(E)L) & E几 1 & EN 2 & En 2 \\
\hline & LoW-68VG| Testing(LIT) & แT 1 & แ⿺辶 2 & แT 3 \\
\hline & 8tekenooder Commitment(3C) & $8 \mathrm{CO}$ & $8 C 2$ & 803 \\
\hline & Tent Organization(TO) & Tol & TOR & TO3 \\
\hline & Testar Proftesalonelam(TPF) & TPF1 & TPFe & TPF3 \\
\hline & & \multicolumn{3}{|c|}{$\begin{array}{c}\text { Level1 } \\
\text { (현재수준) }\end{array}$} \\
\hline
\end{tabular}

Fig. 7. Project Evaluation and Improvement by metrics

테스트 성숙도 모델(TMMi)과 테스트 프로세스 향상 모델 (TPI NEXT)의 상관 연관성 분석을 통해 매핑을 수행하였 다. 이를 통해 두 모델간의 수준별 활동 요소 분석 및 두 모델 간 비/공통요소를 추출하였다. 이는 조직의 테스트 성 숙도를 평가하는 $\mathrm{TMMi}$ 측정만으로도 조직의 테스트 프로 세스를 개선할 수 있는 가이드를 제시하였다. 또한 기존의 테스트 성숙도 상호관련 메트릭[6,8,9] 개선\&확장을 통해, 테 스트 프로세스 성숙도 향상 방안들을 제시하고자 한다. 추 후 TMMi 전체 수준(Level 3 5)과 TPI NEXT의 전체 수 준(Controlled Optimizing)을 상세히 분석하여 확장된 프로 세스 개선 모델을 보완할 예정이다.

\section{참 고 문 헌}

[1] "Test Maturity Model integration(TMMi) Vesrion 3.1", TMMi Foundation, 2012
[2] Bert Linker, Ben Visser "TPI® NEXT: Test Process Improvement improved", www.testingexperience.com.

[3] Ilene. Burnstein, A. Homyen, T. Suwannasart, G. Saxena, and R. Grom, "A Testing Maturity Model for Software Test Process Assessment and Improvement," Software Quality Professional, 1999.

[4] Ilene Burnstein, Taratip Suwannasart, and C.R. Carlson, Developing a Testing Maturity Model: Part I, 1996.

[5] Ilene Burnstein, Taratip Suwannasart, and C.R. Carlson, Developing a Testing Maturity Model: Part II, 1996.

[6] Kidu Kim, R. Youngchul Kim, "A Study on Enhanced Test Maturity Model(TMM) through Test Process Improvement”, Hongik University, 2004.

[7] Kidu Kim, R. Youngchul Kim, "A Study on Enhanced Test Maturity Model(TMM) through Test Process Advancement", KCSE, Vol.1, No.1, 2005.

[8] Kidu Kim, R. Youngchul Kim, "A Study on Enhanced Test Maturity Model with Test Process Improvement", The KIPS Trans D, Vol.14-D, No.1, 2007.

[9] Kidu Kim, R. Youngchul Kim, "A Study of Enhanced Test Maturity Model with Test Process Improvement NEXT", The 37th KIPS Spring Conference, Vol.19, No.1, 2012.

[10] Kidu Kim, R. Youngchul Kim, “Test Process Improvement of Test Organization Through extracting Uncommon/ Common Facts based on mapping TMMi with TPI NEXT", The 38th KIPS Fall Conference, Vol.19, No.2, 2012.

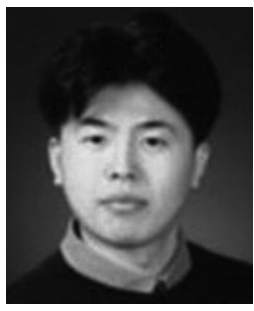

\section{김 기 두}

e-mail :kdkim@tta.or.kr

2003년 홍익대학교 컴퓨터정보통신학부 (학사)

2005년 홍익대학교 소프트웨어공학(석사, 박사수료)

2005년 현 재 한국정보통신기술협회

소프트웨어시험인증연구소 선임연구원

관심분야:TMM\&TMMi, TPI\&TPI NEXT, 테트스프로세스 개선

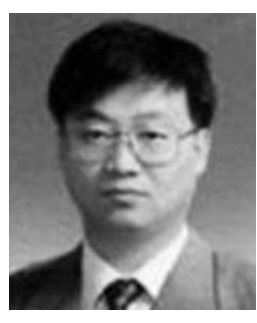

박 용 범

e-mail :ybpark@dankook.ac.kr

1991년 N.Y. Polytechnic University

Science \& Engineering(Ph.D.)

1993년 현 재 단국대학교 컴퓨터과학과 교수

관심분야: Intelligent Software Engineering, Security Software 


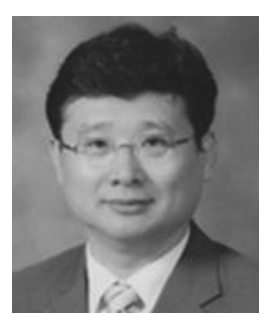

\section{김 영 철}

e-mail : bob@hongik.ac.kr

2000년 일리노이공대 전산과(공학박사)

2000년 2001년 LG 산전 중앙연구소

Embedded system 부장

2001년 현 재 홍익대학교 컴퓨터정보

통신 교수

관심분야: Software Modeling \& Testing, Test Maturity Model (TMM) 